\title{
Idiopathic intracranial hypertension in female homozygous twins
}

\author{
Syouji Fujiwara, Yutaka Sawamura, Tsutomu Kato, Hiroshi Abe, Harumi Katusima
}

Department of

Neurosurgery,

University of

Hokkaido, School of

Medicine, Sapporo,

Japan

S Fujiwara

Y Sawamura

T Kato

H Abe

Department of

Ophthalmology,

Sapporo Medical

University, Sapporo,

Japan

H Katusima

Correspondence to:

Dr Syouji Fujiwara,

Department of

Neurosurgery, University of

Hokkaido School of

Medicine, Kita-15, Nishi-7,

Kita-ku, Sapporo 060, Japan.

Received 10 April 1996

and in revised form

Accepted 2 December 1996

Figure 1 Case 1 (left) and case 2 (right). $A$ direct coronal $C T$ shows thin bony structures at the frontal skull base in both cases. There is an orifice located in the right anterior base of the skull in case 1 (arrow).

\section{Abstract}

The authors report on female homozygous twins with idiopathic intracranial hypertension. At the age of 12 years, both twins simultaneously developed visual disturbances with photophobia. At the age of 19 years, an ophthalmological examination disclosed papilloedema in both their eyes. At the age of 22 years, a lumbar puncture showed raised CSF pressure over $\left(200 \mathrm{~mm} \mathrm{H} \mathrm{H}_{2} \mathrm{O}\right)$ in both twins. Their neurological and radiological examinations were extremely similar; both of them had severely impaired visual acuity and impaired visual field, bilateral optic nerve atrophy, intracranial hypertension, an enlarged and partial empty sella turcica, digital markings of the calvalium, and an enlarged frontal subarachnoid space. This is the first case report describing idiopathic intracranial hypertension occurring in homozygous twins.

$(\mathcal{F}$ Neurol Neurosurg Psychiatry 1997;62:652-654)

Keywords: homozygous twins; CSF rhinorrhoea; intracranial hypertension

Idiopathic intracranial hypertension was first described by Quinke in $1897 .{ }^{1}$ This syndrome

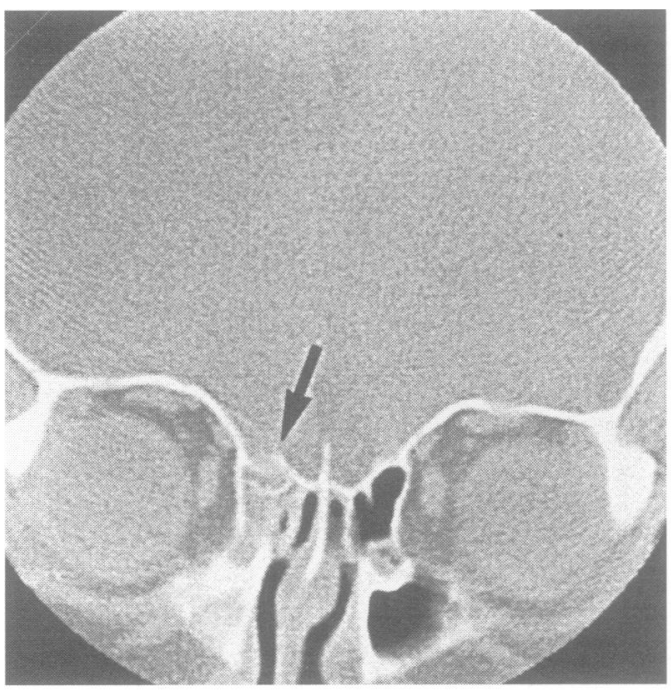

Case 1 is defined as increased intracranial pressure without neurological, laboratory, or radiological evidence of an intracranial space occupying lesion or hydrocephalus. An annual age adjusted incidence rate of idiopathic intracranial hypertension was estimated to be about 0.9 per 100000 population. ${ }^{2}$ Therefore, it is not an uncommon disorder and is most often seen in women of childbearing age either with obesity or recent weight gain. ${ }^{1}$ When obesity was considered the incidence increased to $19 \cdot 3$ per 100000 for women aged 20 to $44 .^{3}$

However, the occurrence of familial intracranial hypertension is rare. To our knowledge, only 10 case reports concerning familial intracranial hypertension have been reported in the literature. ${ }^{14-12}$ The disorder was found in two pairs of heterozygous twins previously, ${ }^{510}$ but not in homozygous twins. This paper describes idiopathic intracranial hypertension occurring in female homozygous twins.

\section{Case reports}

CASE 1

A 22 year old woman was referred to our hospital with a 10 month history of watery nasal discharge. The patient was born in 1973 as a homozygous twin. The delivery was six weeks premature. At the age of 12 years, she experienced photophobia and developed progressive loss of visual acuity. The corrected visual acuity

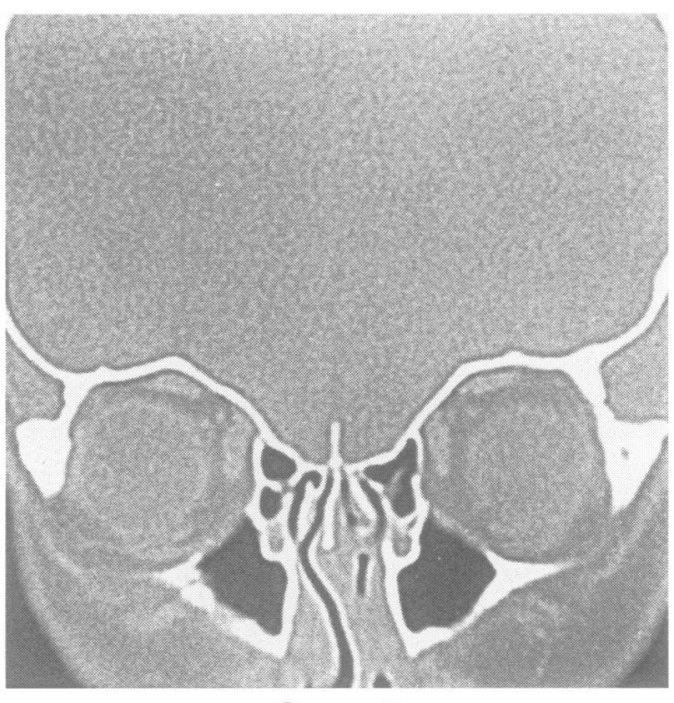

Case 2 
was 0.07 in the right eye and 0.2 in the left eye. At the age of 19 years, an ophthalmological examination disclosed papilloedema and visual field defects in both eyes. At the age of 21 years, a watery nasal discharge from the right side of the nose occurred without any history of head injury, surgical procedure, or sinus disease.

On admission, the patient was well nourished, but not obese. She had had no menstrual irregularity, oral contraceptive use, pregnancy, or thyroid disease. And she had no headache. An ophthalmological examination disclosed optic atrophy in both eyes with decreased visual acuity; corrected acuities were 0.06 in the right eye and 0.09 in the left eye. A skull radiograph showed digital markings on the calvarium. Direct coronal CT (fig 1) showed a tiny orifice in the right anterior fossa and clouded ethmoidal sinus. Sagittal MRI showed an enlarged and partially empty sella (fig 2). Axial T2 weighted MRI showed wide subarachnoid spaces in the frontal region and minimally enlarged lateral ventricles (fig 2).

Figure 2 Case 1 (left). and case 2 (right). Brain MRI shows a partially empty sella and a slight enlargement of the bilateral frontal subarachnoid space in both cases.

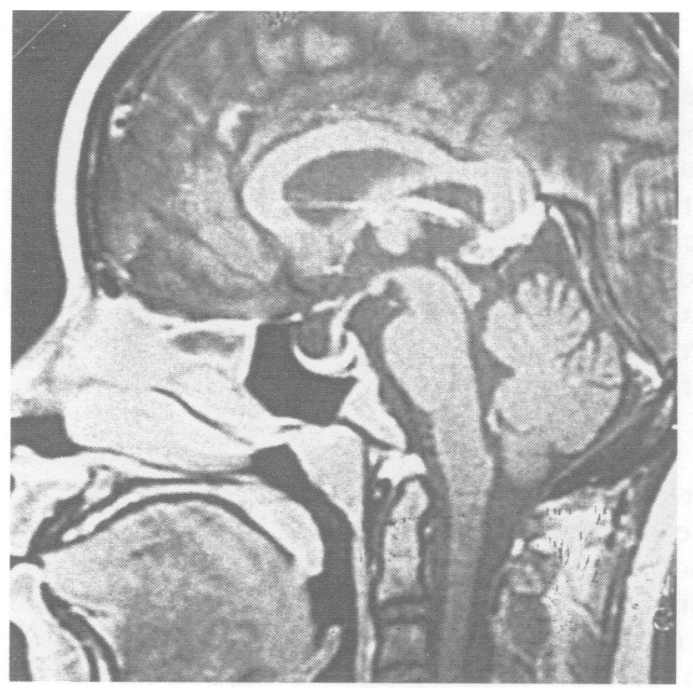

Case 1

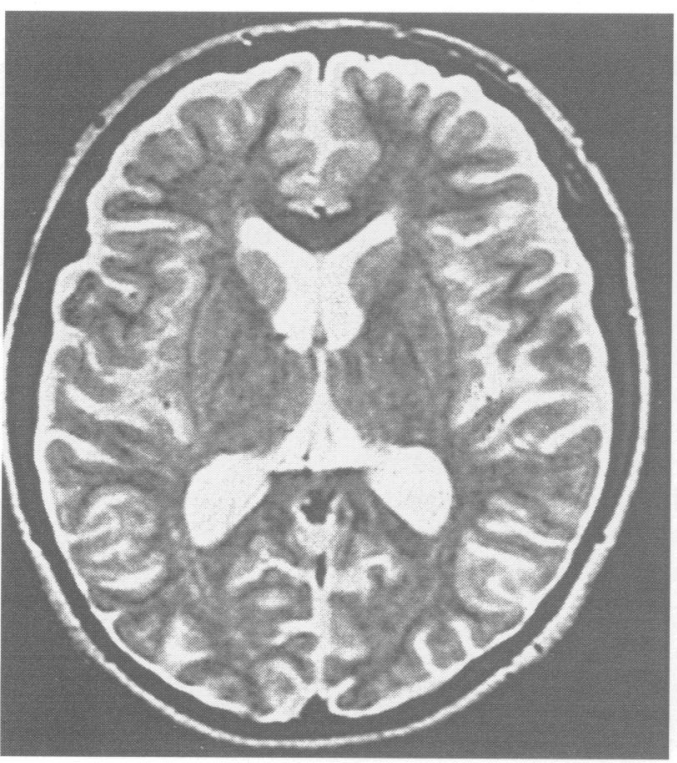

Case 1
The patient underwent a bifrontal craniotomy through the right frontal skull base to repair the CSF leakage. After surgery the rhinorrhoea ceased, but headache, nausea, and vomiting gradually developed. A lumbar puncture showed a high opening pressure of 340 $\mathrm{mm} \mathrm{H}_{2} \mathrm{O}$ with normal cell count and normal concentrations of glucose, protein, albumin, prealbumin, and $\gamma$-immunoglobulin. Because of the high intracranial pressure, a ventricleperitoneal shunt was placed. The patient's clinical course after the surgery was uneventful and the visual function remained stable.

CASE 2

The twin sister of this patient was also seen at the age of 22 years. A blood type analysis confirmed that they were homozygous twins. She presented a nearly identical clinical history to her sister. At the age of 12 years, she experienced photophobia and developed a progressive loss of visual acuity; the corrected visual acuities were 0.04 in both eyes. At the age of 19 years, an ophthalmological examination disclosed papilloedema and visual field defects

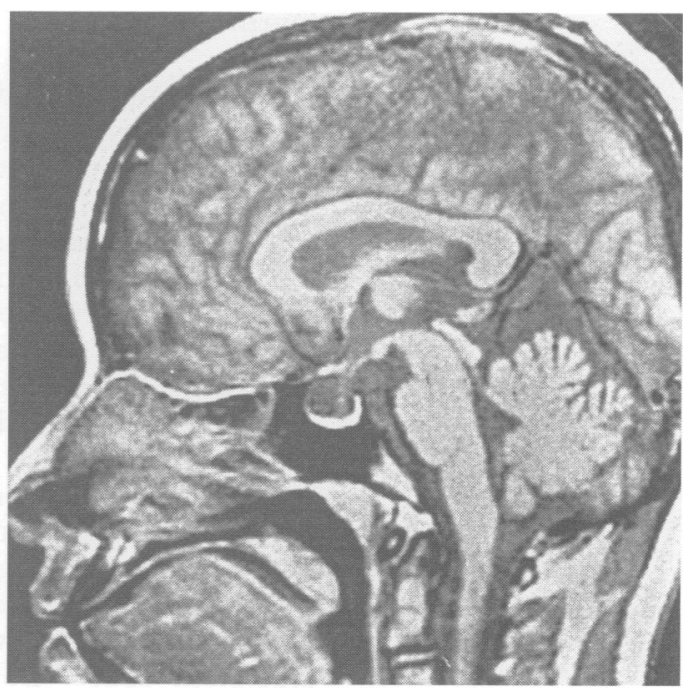

Case 2

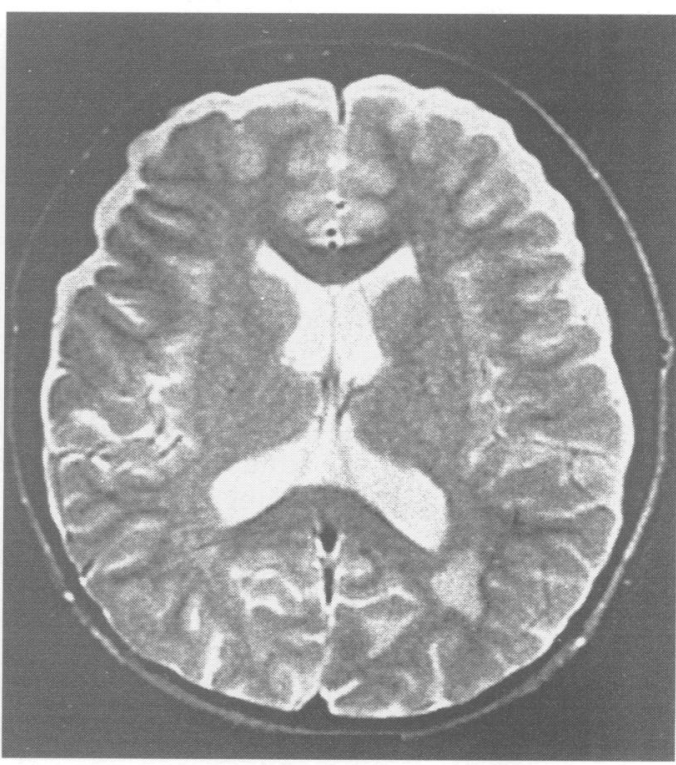

Case 2 
in both eyes. Like her sister, she was not obese and had had no menstrual irregularity, oral contraceptive use, pregnancy, thyroid disease, or headache. An ophthalmological examination showed optic atrophy in both eyes with decreased visual acuity; corrected acuities were 0.04 in both eyes. A lumbar puncture showed a pressure of $210 \mathrm{~mm} \mathrm{H}_{2} \mathrm{O}$. The CSF study was normal. A skull radiograph showed digital markings on the calvalium. A direct coronal CT (fig 1) showed thin bony structures in the anterior skull base and deep olfactory grooves. Sagittal MRI showed an enlarged and partially empty sella (fig 2). An axial T2 weighted MRI showed wide subarachnoid spaces in the frontal region and minimally enlarged lateral ventricles (fig 2). The patient underwent a ventricle-peritoneal shunt operation. Lately she has been free from deterioration of visual functions.

\section{Discussion}

Idiopathic intracranial hypertension is a condition that occurs predominantly in women of childbearing age with obesity and recent weight gain; there is a peak incidence in the fourth decade. ${ }^{9}$ Neither of our patients were obese.

The confirmed diagnosis of idiopathic intracranial hypertension requires (1) a documented increase in intracranial pressure, more than $200 \mathrm{~mm} \mathrm{H}_{2} \mathrm{O}$ in the non-obese patient; (2) a normal neurological examination, except for papilloedema with deficits of visual acuity and an occasional abducens nerve palsy; (3) the absence of a space occupying lesion or ventricular enlargement, except for an empty sella on CT or MRI; and (4) normal CSF composition. ${ }^{13} 1415$

Our cases are consistent with these criteria. Although the sizes of the lateral ventricles were slightly large, a diagnosis of hydrocephalus was equivocal. In addition, the MRI showed minimal enlargement of the bifrontal subarachnoid space. Both patients might have developed an empty sella because of high intracranial CSF pressure for several years. Slight enlargements of the lateral ventricles, the sella, and the frontal subarachnoid space are not uncommon radiological findings for idiopathic intracranial hypertension..$^{591316}$

Johnston and Morgan ${ }^{6}$ state that familial intracranial hypertension supports the idea of dysfunctional CSF absorption in this disease. Because of the relatively late onset of intracranial hypertension, Cardoso et al postulate that age might account for disturbed CSF absorption. In infants, impaired CSF circulation is more likely to dilate the ventricles but in adults, complete myelination and mature brains resist ventricle dilatation. So intracranial hypertension occurs after a long asymptomatic history and without ventriculomegaly.
Ten previous case reports have described familial idiopathic intracranial hypertension. The cases include one mother and son, ${ }^{8}$ a mother and two of four daughters and a son, ${ }^{6}$ three mother-daughter pairs, ${ }^{7912}$ three pairs of siblings, ${ }^{1411}$ and two pairs of heterozygous twins. ${ }^{510}$

To our knowledge, ours is the first report of idiopathic intracranial hypertension occurring in homozygous twins, and the findings support the hypothesis of genetic transmission of this disease.

The simultaneous occurrence of visual disturbance in the twins is interesting. Torlai et al also reported male twins who became symptomatic simultaneously and followed similar clinical courses for more than two years. ${ }^{10} \mathrm{We}$ think that the intracranial hypertension was not present in early childhood in our patients, but that it gradually developed at the beginning of their adolescence. Many studies have emphasised the association of idiopathic intracranial hypertension with menstrual irregularities, pregnancy, oral contraceptive use, and a heterogeneous group of endocrine diseases. ${ }^{21819}$ Although we have not investigated hormonal concentrations in our patients, the aetiology of idiopathic intracranial hypertension may relate to a balance of secretion of gonadotrophin(s).

1 Howe JG, Saunders M, Clarke P. Familial benign intracranial hypertension. Acta Neurochir(Wien) 1973;29:173-5.

2 Radhakrishnan K, Ahlskog JE, Cross SA, Kurland LT, O'Fallon WM. Idiopathic intracranial hypertension (pseudotumor cerebri). Arch Neurol 1993;50:78-80.

3 Durcan FJ, Corbett JJ, Wall M. The incidence of pseudotumor cerebri. Population studies in Iowa and Louisiana. Arch Neurol 1988;45:875-7.

4 Buchheit WA, Burton C, Haag B, Shaw D. Papilledema and idiopathic intracranial hypertension. A report of a and idiopathic intracranial hypertension. A report

5 Gardner K, Cox T, Digre KB. Idiopathic intracranial hypertension associated with tetracycline use in fraternal hypertension associated with tetracycline use in fraternal
twins. Case reports and review. Neurology 1995;45:6-10.

6 Johnston I, Morgan MK. A Familial Coincidence Of Pseudotumor Cerebri and Communicating hydrocephalus. Neurosurgery 1991;28:727-9.

7 Kharode C, McAbee G, Sherman J, Kaufman M. Familial Intracranial Hypertension. Report of a case and review of the literature. F Child Neurol 1992;7:196-8.

8 Rothner AD, Brust JCM. Pseudotumor cerebri. Report of a familial occurrence. Arch Neurol 1974;30:110-1.

9 Shapiro I, Shapiro SK. Familial pseudotumor cerebri and the empty sella syndrome. Ann Ophthalmol 1980;9: $1045-8$.

10 Torlai F, Galassi G, Debbia A, Crisi G, Peduzzi M. Familial pseudotumor cerebri in male heterozygous Familial pseudotumor cerebri

11 Traviesa DC, Schwartzman RJ, Glaser JS, Savino P. Familial benign intracranial hypertension. $\mathscr{f}$ Neurol Familial benign intracranial hype
Neurosurg Psychiatry 1976;39:420-3.

12 Venable HP. Pseudotumor cerebri. Further studies. $f$ Natl Med Assoc 1973;65:194-7.

13 Marcelis J, Silberstein SD. Idiopathic intracranial hypertension without papilledema. Arch Neurol 1991;48:392-9.

14 Smith $J$. Whence pseudotumor cerebri. $f$ Clin Neuro Ophthalmol 1985;5:55-6.

15 Wall $M$, George D. Idiopathic intracranial hypertension: a prospective study of 50 patients. Brain 1991;114:155-80

6 Radhakrishnan K, Ahlskog JE, Garrity JA, Kurland LT Idiopathic intracranial hypertension. Mayo Clin Proc 1994;69:169-80.

17 Cardoso ER, Del Bigio MR, Schroeder G. Age-dependent changes of cerebral ventricle size. Part 1 . Review of changes of cerebral ventricle size. Part 1. Review of
intracranial fluid collections. Acta Neurochir (Wien) 1989;
97:40-6.

18 Donaldson JO. Pathogenesis of pseudotumor cerebri syndromes. Neurology 1981;31:877-80.

19 Fishman RA. The pathophysiology of pseudotumor cerebri: an unsolved puzzle. Arch Neurol 1984;41:257-8. 